\title{
A simple and efficient method for isomorphism identification of planar kinematic chains
}

Luchuan Yu ( $\nabla$ yulcsdu@foxmail.com )

Wenzhou University https://orcid.org/0000-0002-3905-1513

Chenxu Cai

Shandong University

Jianhua Zhang

Shandong University

Qinhe Zhang

Shandong University

\section{Research Article}

Keywords: isomorphism identification, adjacency matrix, pseudo-independence loop, kinematic chain

Posted Date: June 7th, 2021

DOl: https://doi.org/10.21203/rs.3.rs-470798/v1

License: (c) (1) This work is licensed under a Creative Commons Attribution 4.0 International License. Read Full License 


\title{
A simple and efficient method for isomorphism identification of planar kinematic chains
}

Luchuan Yu', a Chenxu Cai ${ }^{2,3, \mathrm{~b}}$, Jianhua Zhang ${ }^{2,3, \mathrm{c}}$, Qinhe Zhang ${ }^{2,3, \mathrm{~d}}$

1 College of Mechanical and Electrical Engineering, Wenzhou University, Wenzhou 325035, PR China

2 Key Laboratory of High Efficiency and Clean Mechanical Manufacture (Ministry of Education of the

People's Republic of China), School of Mechanical Engineering, Shandong University, Jinan 250061, China

3 National Demonstration Center for Experimental Mechanical Engineering Education (Shandong University), School of Mechanical Engineering, Shandong University, Jinan 250061, China

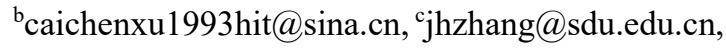

\author{
dzhangqh@sdu.edu.cn \\ $\mathrm{a}^{*}$ Corresponding author: Luchuan $\mathrm{Yu}$ \\ Email: yulcsdu@foxmail.com
}

Abstract: Isomorphism identification plays an important role in structural design and innovative design. Based on the adjacency matrix and loop theory, a new method is proposed in this paper to identify the isomorphic kinematic chains. It enriches the application of loop-based theory for isomorphism identification. In the kinematic chain, links and joints are connected alternatively and every link corresponds to a fixed link degree. Due to the inherent characteristics, the labeled sequence of links can be random, which does not affect the result of isomorphism identification. By the programming software MATLAB, some examples with 6-, 8-, 10-, 11-, 12-link kinematic chains, and 15-vertex topological graphs are presented. Results show that the proposed method applies to topology graphs and kinematic chains with one or multiple joints. Compared with other methods, the proposed method is confirmed correctly. And there is no counterexample. It lays a solid foundation for structural synthesis in the future.

Keywords: isomorphism identification; adjacency matrix; pseudo-independence loop; kinematic chain

\section{Introduction}

Application of isomorphism identification to structural synthesis has been proven to be an effective procedure, which has a large effect on the efficiency and accuracy of structural synthesis. Up to now, there have been many valuable methods illustrated to solve the isomorphism problem.

Methods based on the adjacency matrix and incidence matrix have been widely used in the field. Among many others, He et al. $(2001,2003)$ proposed the method, which is based on the adjacency matrix and makes use of the information of the eigenvalue and eigenvector, which is called eigensystem approach. They further proposed a modified adjacency matrix called adjusted adjacency matrix in order to study a sufficient condition for the quadratic form approach (He et al., 2005). Yang et al. (2012) proposed a method based on the incident matrix. Based on the link-link shortest path distance, Madan (2014) used a kinematic chain label as the unique identifier for isomorphism identification among kinematic chains.

The loop-based method is an effective way to deal with the problem. The application of the loop-based technique in association with the hamming number (Pathapati and Rao 2002; Dharanipragada and Chintada 2016) was introduced to investigate and identify the isomorphism problem. Meanwhile, Rao and Pathapati (2000) developed a method based on the loop to reveal simultaneously that chain and link are isomorphic respectively. Based on the concept of the independent loop set and a new structure decomposition 
algorithm, the edge-based loop algebra theory (Ding, Zhao, and Huang 2010; Ding and Huang 2007) was presented to detect the isomorphism.

The application of intelligent algorithms has been focused on. Kong et al. (1999) provided an artificial neural network technique to identify the isomorphism of the mechanism in the kinematic chain. A new method based on novel evolutionary approaches was used in isomorphism identification, which included artificial immune system (Xiao, Tao, and Liu 2005) and ant algorithm (Yang et al. 2007). To improve the efficiency and reliability of isomorphism identification, Yang and Zeng (2009) demonstrated an adaptive hybrid genetic algorithm by mixing the improved genetic algorithm and local search algorithm.

There exist other streams of studies on isomorphism identification. After mechanism topologies were summarised and divided into four abstraction levels, Zhang and Li (1999) developed Vertex Feature Degree code approach to compare mechanism topology for all levels. And the proposed code approach was shown to be applicable to the whole problem space of mechanism topology identification. Arvind et al. (2015) presented Boolean functions to study the complexity of isomorphism testing. The functions were illustrated by decision trees or decision lists. Based on the concept of information entropy, Rai and Punjabi (2018) introduced the formulae of power and efficiency to detect the isomorphism in the kinematic chain.

In the above literatures, the approaches are very effective to solve the problem in their papers. Meanwhile, the automatic method for isomorphism identification is favoured by researchers at home and abroad. By the programming software MATLAB, this paper proposes a new method based on the adjacency matrix and pseudo-independence loop to detect the isomorphic mechanisms.

The rest of the paper is divided as follows. In section 2, some common terminologies and principles are defined. In section 3, the detailed steps for isomorphism identification of kinematic chains and the complexity of the proposed method are illustrated. In section 4 , some examples are employed to verify the correctness of the proposed method. In section 5, a discussion is demonstrated. Finally, section 6 is reserved for conclusions.

\section{Common terminologies}

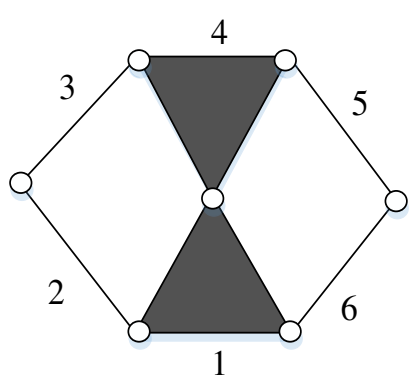

(a)

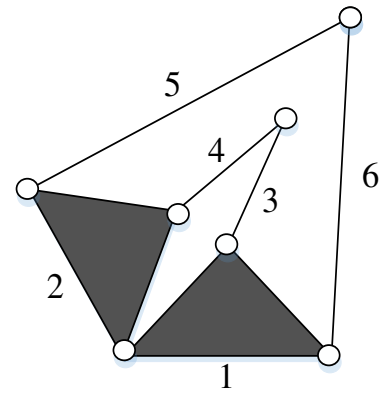

(b)

Fig. 1 Simple kinematic chains with six links

Consider simple kinematic chains with six links, shown in Fig. 1. They are used to illustrate common terminologies clearly. 


\subsection{Basic concepts}

\subsubsection{Pseudo-independence loop (PIL)}

In this paper, the home link is defined as the starting link of the closed loop. For example, the loop (1, 2, 3, 4, 1) in Fig. 1(a) is a closed loop. Link 1 is the home link. The inner loop of the kinematic chain inside which there is no other closed loop is known as the independent loop. For the simple planar kinematic chain in Fig. 1(a), the independent loop is two. However, PIL and independent loop are different concepts. PIL is the closed loop starting from the home link and finally back to the home link. It may contain one or more independent loops in its inner loop.

For example, if link 1 is selected as a home link, there will be three PILs in Fig. 1(a). They are the loops $(1,2,3,4,1),(1,2,3,4,5,6,1)$ and $(1,4,5,6,1)$ respectively. If the link 2 is selected as a home link, there will be two PILs in Fig. 1(a). They are the loops $(2,3,4,1,2)$ and $(2,3,4,5,6,1,2)$ respectively. However, there are two independent loops in Fig. 1(a) whatever the home link is. The selection of home links does not affect the number of independent loops. Besides, the direction and sequence of labeled links do not affect PIL. The loops $(1,2,3,4,1)$ and $(1,4,3,2,1)$ are equivalent in this paper. And there is no repeated labeled link except home link in a PIL.

Link degree represents the link type. The degrees of binary link, ternary link, quaternary link are 2, 3, 4 respectively. If link 1 in Fig. 1(a) is selected as the home link, PILs can be changed into (3, 2, 2, 3, 3), (3, $3,2,2,3),(3,2,2,3,2,2,3)$, which correspond to PILs $(1,2,3,4,1),(1,4,5,6,1)$ and $(1,2,3,4,5,6,1)$ respectively.

In addition to the definition of the independent loop, there have been many other classic definitions related to the loop, such as linkage path code (Yan and Hwang 1984) and self-returning link-walk (Yadav, Pratap, and Agrawal 1996). Compared with the classic definitions, PIL is a closed loop with a finite orderly sequence of link numbers or link degrees only instead of including joint numbers. PIL in the form of the link degree can reflect the link type.

\subsubsection{Adjacency matrix (AM)}

A kinematic chain can be represented by a link-link $A M$. The number of columns in the $A M$ is equal to that of rows. They are determined by the number of links. If link $i$ and link $j$ are connected by a vertex, the value of $A M_{i j}$ or $A M_{j i}$ will be one. Otherwise, it will be zero. Therefore, the expression of $A M_{i j}$ is defined as

$$
A M_{i j}=\left\{\begin{array}{l}
1, \text { if link } i \text { and link } j \text { are adjacent directly } \\
0, \text { otherwise }
\end{array}\right.
$$

\subsection{Basic principles}

2.2.1 Principle 1: For isomorphism identification in the kinematic chains, home links should be the same.

The home link plays an important role in the PIL. The same links are links with identical link degrees. Erroneous results can occur if selected home links are different. Consider simple kinematic chains with 
six links in Fig. 1. For every possible home link, PILs and corresponding link degree sequences are shown in Table 1 and Table 2.

Table 1. PILs of the kinematic chain in Fig. 1(a)

\begin{tabular}{cl}
\hline Home link & PIL and corresponding link degree array \\
\hline 1 & $(1,2,3,4,1),(1,2,3,4,5,6,1),(1,4,5,6,1)$ \\
& $(3,2,2,3,3),(3,2,2,3,2,2,3),(3,3,2,2,3)$ \\
& $(2,3,4,1,2),(2,3,4,5,6,1,2)$ \\
& $(2,2,3,3,2),(2,2,3,2,2,3,2)$ \\
& $(3,4,1,2,3),(3,4,5,6,1,2,3)$ \\
& $(2,3,3,2,2),(2,3,2,2,3,2,2)$ \\
& $(4,1,2,3,4),(4,5,6,1,4),(4,5,6,1,2,3,4)$ \\
& $(3,3,2,2,3),(3,2,2,3,3),(3,2,2,3,2,2,3)$ \\
& $(5,6,1,4,5),(5,6,1,2,3,4,5)$ \\
& $(2,2,3,3,2),(2,2,3,2,2,3,2)$ \\
& $(6,1,4,5,6),(6,1,2,3,4,5,6)$ \\
& $(2,3,3,2,2),(2,3,2,2,3,2,2)$ \\
\end{tabular}

Table 2. PILs of the kinematic chain in Fig. 1(b)

\begin{tabular}{cl}
\hline Home link & PIL and corresponding link degree array \\
\hline 1 & $(1,2,4,3,1),(1,3,4,2,5,6,1),(1,2,5,6,1)$ \\
& $(3,3,2,2,3),(3,2,2,3,2,2,3),(3,3,2,2,3)$ \\
2 & $(2,4,3,1,2),(2,5,6,1,3,4,2),(2,5,6,1,2)$ \\
& $(3,2,2,3,3),(3,2,2,3,2,2,3),(3,2,2,3,3)$ \\
3 & $(3,4,2,1,3),(3,4,2,5,6,1,3)$ \\
& $(2,2,3,3,2),(2,2,3,2,2,3,2)$ \\
& $(4,2,1,3,4),(4,2,5,6,1,3,4)$ \\
& $(2,3,3,2,2),(2,3,2,2,3,2,2)$ \\
& $(5,6,1,2,5),(5,6,1,3,4,2,5)$ \\
& $(2,2,3,3,2),(2,2,3,2,2,3,2)$ \\
& $(6,1,2,5,6),(6,1,3,4,2,5,6)$ \\
& $(2,3,3,2,2),(2,3,2,2,3,2,2)$
\end{tabular}

In Fig. 1, there will be three PILs in each kinematic chain if link 1 is selected as the home link. There will be two PILs if link 4 in Fig. 1(b) is selected as the home link. However, the erroneous result can occur if link 1 in Fig. 1(a) and link 4 in Fig. 1(b) are both selected as the home links. In this paper, link 1 is defined as the home link in the kinematic chains.

Principle 1 will not come into effect until link assortments in the kinematic chains are identical. Otherwise, it will be meaningless to select the home link even if PIL sets in the form of the link degree in the kinematic chains are one-to-one correspondences. 


\subsubsection{Principle 2: Each labeled link except the home link appears only once in a PIL.}

Principle 2 can guarantee that PIL is a closed loop with a finite orderly sequence of link numbers or link degrees. It's very convenient to reflect the characteristics of the PIL, which include link type and link sequence. If principle 2 is ignored, it will lead to the infinite sequence of PIL with relabeled links. Based on principle 2, it is not only beneficial to detect the isomorphic kinematic chains but also applicable to automatic sketching of kinematic chains (Bedi and Sanyal 2013).

The body-fractionated kinematic chain contains at least one body that divides the chain into several non-fractioned kinematic chains. For the non-fractionated kinematic chain, links except home link can appear only once in a PIL. For the planar body-fractionated kinematic chain, it is difficult to find all PILs covering all independent loops when the non-fractionated link is selected as the home link. Therefore, it's important to select the home link in the body-fractionated kinematic chain or divide the body-fractionated kinematic chain into several non-fractioned kinematic chains properly.

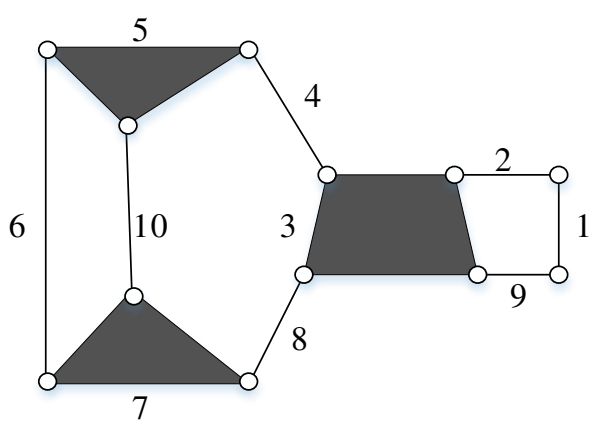

(a)

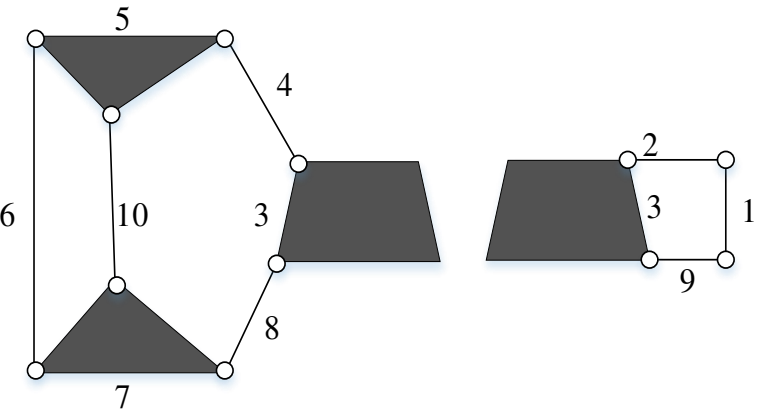

(b)

Fig. 2 Fractionated kinematic chain

In Fig. 2(a), there is a loop $(1,2,3,4,5,6,7,8,3,9,1)$. However, the loop doesn't meet principle 2. For a fractionated kinematic chain, the home link is usually the fractionated link. To meet the principle 2, link 3 in Fig. 2(a) is selected as the home link, or fractioned kinematic chain in Fig. 2(a) is transformed into two non-fractioned kinematic chains in Fig. 2(b). Under this circumstance, the proposed method is still applicable.

\section{Method}

\subsection{The method for isomorphism identification}

The proposed method is based on the $A M$ and PIL of the kinematic chain. Under the principle 1 and principle 2, the detailed steps are:

Step 1: Define the home link.

The kinematic chain in Fig. 1(a) should be labeled. Due to the inherent relations among links and vertices, the labeled sequence of links can be random. Link 1 in Fig. 1(a) is selected as the home link. The selection of the home link in Fig. 1(b) should be consistent with that in Fig. 1(a). There is no requirement for other links. For the kinematic chains in Fig. 1, the labeled sequence of other links is clockwise. 
Step 2: Obtain AMs.

The AMs in Fig. 1(a) and Fig. 1(b) are

$$
A M_{a}=\left[\begin{array}{cccccc}
0 & 1 & 0 & 1 & 0 & 1 \\
1 & 0 & 1 & 0 & 0 & 0 \\
0 & 1 & 0 & 1 & 0 & 0 \\
1 & 0 & 1 & 0 & 1 & 0 \\
0 & 0 & 0 & 1 & 0 & 1 \\
1 & 0 & 0 & 0 & 1 & 0
\end{array}\right] \quad A M_{b}=\left[\begin{array}{llllll}
0 & 1 & 1 & 0 & 0 & 1 \\
1 & 0 & 0 & 1 & 1 & 0 \\
1 & 0 & 0 & 1 & 0 & 0 \\
0 & 1 & 1 & 0 & 0 & 0 \\
0 & 1 & 0 & 0 & 0 & 1 \\
1 & 0 & 0 & 0 & 1 & 0
\end{array}\right]
$$

Step 3: Find all PILs in the form of the link degree.

PILs in Fig. 1(a) are $(1,2,3,4,1),(1,4,5,6,1)$ and $(1,2,3,4,5,6,1)$. The corresponding PILs in the form of the link degree are $(3,2,2,3,3),(3,3,2,2,3),(3,2,2,3,2,2,3)$. Similarly, PILs in Fig. 1(b) are $(1,2,4,3,1),(1,2,5,6,1),(1,3,4,2,5,6,1)$. And the corresponding PILs in the form of the link degree are $(3,3,2,2,3),(3,3,2,2,3),(3,2,2,3,2,2,3)$.

Step 4: Isomorphism identification.

If PIL sets in the form of the link degree in the kinematic chains are one-to-one correspondences, kinematic chains in Fig. 1 are isomorphic. In fact, they are isomorphic.

\subsection{The procedures to obtain PILs}

It's a crucial part to obtain all PILs in the kinematic chain. Take the kinematic chain in Fig. 1(a) as an example. In this section, the procedures to obtain PILs are shown. It's also applicable to the program.

Step 1: The PIL starts from the home link whose labeled number is 1 . As seen in $A M_{a}$ in section 3.1, there are three rows whose numbers are 1 in the first column. It means the home link is connected with the other three links. Therefore, the first two labeled numbers in the PILs are $(1,2),(1,4)$, and $(1,6)$.

Step 2: Similarly, based on principle 2, the third labeled number is 3 in the PIL whose first two labeled number is $(1,2)$. And the third labeled number is 5 in the PIL whose first two labeled number is $(1,6)$. However, for the PIL whose first two labeled number is $(1,4)$, the third labeled number can be 3 or 5 . Under this circumstance, there are two PILs that are $(1,4,3)$ and $(1,4,5)$ respectively. Therefore, there are four PILs. They are $(1,2,3),(1,4,3),(1,4,5)$ and $(1,6,5)$.

Step 3: Repeat step 2 until the labeled number of link 1 arises again. If step 2 is executed twice, PILs will be changed into $(1,2,3,4),(1,4,3,2),(1,4,5,6)$ and $(1,6,5,4)$. If step 2 is executed three times, PILs will be changed into $(1,2,3,4,1),(1,2,3,4,5),(1,4,3,2,1),(1,4,5,6,1),(1,6,5,4,1)$ and $(1,6$, $5,4,3)$. Up to now, there have been four PILs whose first number and last number are both 1 . In other words, they are closed loops.

Step 4: It's essential to detect and delete the isomorphic loops in the step. There are 6 PILs after step 3. They are $(1,2,3,4,1),(1,2,3,4,5,6,1),(1,4,3,2,1),(1,4,5,6,1),(1,6,5,4,1)$ and $(1,6,5,4,3,2$, 1). Because PILs start from and end in the home link. The only difference for the isomorphic loops is the labeled direction. The PIL (1, 2, 3, 4, 5, 6, 1) and the PIL (1, 6, 5, 4, 3, 2, 1) are isomorphic loop. After step 4, there will be 3 PILs. They are $(1,2,3,4,1),(1,4,5,6,1)$ and $(1,2,3,4,5,6,1)$.

Therefore, the whole flow chart of the proposed method is shown in Fig. 3. 


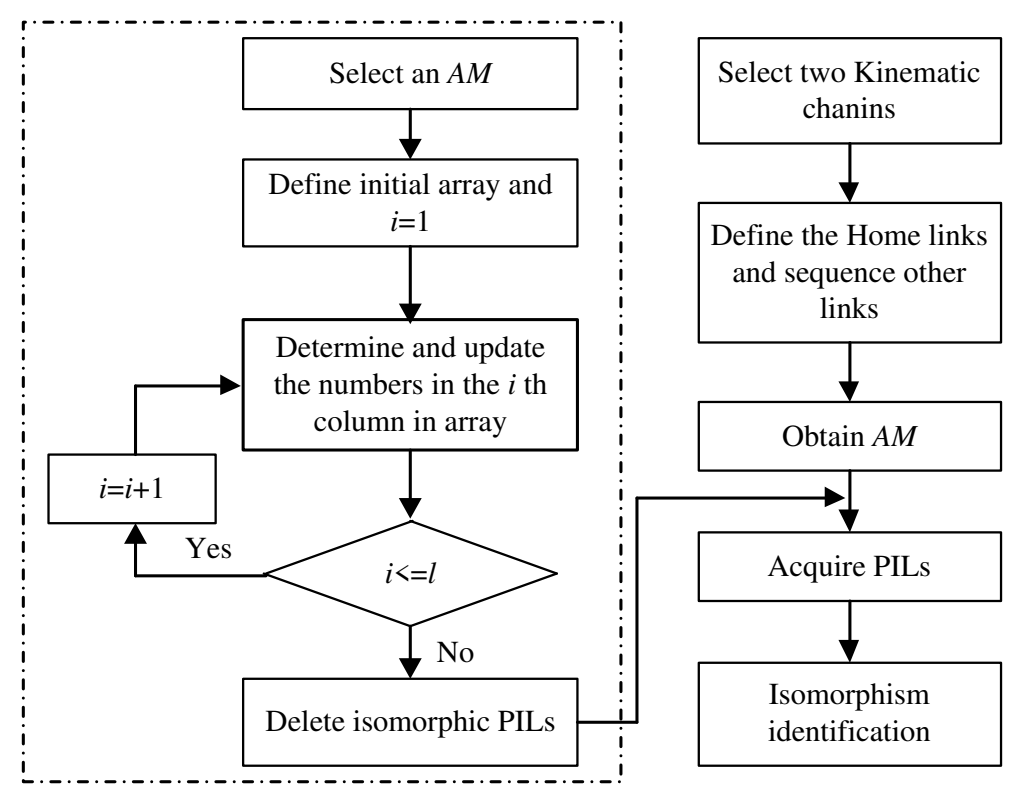

Fig. 3 The flowchart of the proposed method

\subsection{The complexity analysis of the proposed method}

Erroneous results can occur if selected home links are different. In the worst situation, if no information is provided, the home link must be defined in all possible different ways. There are two n-link kinematic chains $a$ and $b$. Assume one link in the kinematic chain $a$ is selected as the home link. For kinematic chain $b$, the maximum number of comparisons among different home links to be made is $n$, and the number of PILs for a home link is $m$. When home links between kinematic chains are identical, time consumption is basically dedicated to obtaining PILs and checking whether there are one-to-one mappings between PIL sets. If there are at most $m$ PILs in the kinematic chain, comparisons among PIL sets will be at most $m(m+1) / 2$. Therefore, the maximum comparison of the proposed method is $m n(m+1) / 2$.

However, the selection of the home link can be restricted by the link assortment (binary link, ternary link, and so on), which can effectively reduce the computation of the comparison. The actual comparison is much less than $m n(m+1) / 2$.

\section{Application of the proposed method for isomorphism identification}

There is a clear discriminant criterion and standardized procedures of the proposed method. It's very convenient to program in the software MATLAB. The flow chart in Fig. 3 is illustrated in two main procedures. It consists of obtaining PILs and isomorphism identification.

\subsection{Example}

Consider a set of kinematic chains with eight links (Madan 2014; Kamesh, Rao, and Rao 2017). It is shown in Fig. 4. 


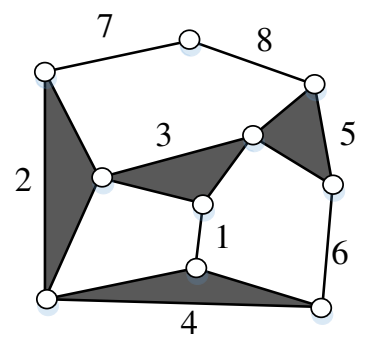

(a)

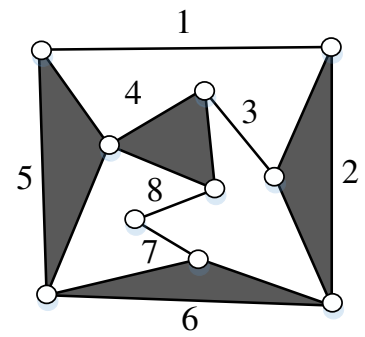

(b)

Fig. 4 The kinematic chain with eight links

The $A M s$ of kinematic chains in Fig. 4(a) and Fig. 4(b) are represented by $A M_{a}$ and $A M_{b}$ respectively.

$$
A M_{a}=\left[\begin{array}{llllllll}
0 & 0 & 1 & 1 & 0 & 0 & 0 & 0 \\
0 & 0 & 1 & 1 & 0 & 0 & 1 & 0 \\
1 & 1 & 0 & 0 & 1 & 0 & 0 & 0 \\
1 & 1 & 0 & 0 & 0 & 1 & 0 & 0 \\
0 & 0 & 1 & 0 & 0 & 1 & 0 & 1 \\
0 & 0 & 0 & 1 & 1 & 0 & 0 & 0 \\
0 & 1 & 0 & 0 & 0 & 0 & 0 & 1 \\
0 & 0 & 0 & 0 & 1 & 0 & 1 & 0
\end{array}\right] \quad A M_{b}=\left[\begin{array}{llllllll}
0 & 1 & 0 & 0 & 1 & 0 & 0 & 0 \\
1 & 0 & 1 & 0 & 0 & 1 & 0 & 0 \\
0 & 1 & 0 & 1 & 0 & 0 & 0 & 0 \\
0 & 0 & 1 & 0 & 1 & 0 & 0 & 1 \\
1 & 0 & 0 & 1 & 0 & 1 & 0 & 0 \\
0 & 1 & 0 & 0 & 1 & 0 & 1 & 0 \\
0 & 0 & 0 & 0 & 0 & 1 & 0 & 1 \\
0 & 0 & 0 & 1 & 0 & 0 & 1 & 0
\end{array}\right]
$$

After the procedures in section 3.2 are executed, the number of PILs in Fig. 4(a) is four. PILs include $(1,3,2,4,1),(1,3,5,6,4,1),(1,3,5,8,7,2,4,1)$ and $(1,3,2,7,8,5,6,4,1)$. PILs in the form of the link degree are $(2,3,3,3,2),(2,3,3,2,3,2),(2,3,3,2,2,3,3,2)$ and $(2,3,3,2,2,3,2,3,2)$.

In Fig. 4(b), the number of PILs is also four. PILs include $(1,2,3,4,5,1),(1,2,6,5,1),(1,2,3,4,8$, $7,6,5,1)$ and $(1,2,6,7,8,4,5,1)$. And PILs in the form of the link degree are $(2,3,2,3,3,2),(2,3,3,3$, 2), (2, 3, 2, 3, 2, 2, 3, 3, 2) and (2, 3, 3, 2, 2, 3, 3, 2).

Based on the discriminant criterion, kinematic chains in Fig. 4 are isomorphic.

\subsection{Example}

Consider a set of kinematic chains with ten links (Kamesh, Rao, and Rao 2017; Dargar, Hasan, and Khan 2011). It is shown in Fig. 5.

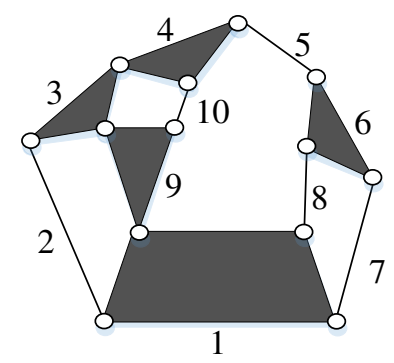

(a)

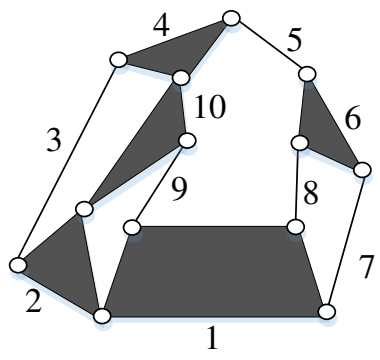

(b)

Fig. 5 The kinematic chain with ten links

The $A M s$ of kinematic chains in Fig. 5(a) and Fig. 5(b) are represented by $A M_{a}$ and $A M_{b}$ respectively. 
After the procedures in section 3.2 are executed, the number of PILs in Fig. 5(a) and that in Fig. 5(b) are both eleven. They are illustrated in Table 3. Based on the discriminant criterion, the two kinematic chains are isomorphic.

$$
A M_{a}=\left[\begin{array}{cccccccccc}
0 & 1 & 0 & 0 & 0 & 0 & 1 & 1 & 1 & 0 \\
1 & 0 & 1 & 0 & 0 & 0 & 0 & 0 & 0 & 0 \\
0 & 1 & 0 & 1 & 0 & 0 & 0 & 0 & 1 & 0 \\
0 & 0 & 1 & 0 & 1 & 0 & 0 & 0 & 0 & 1 \\
0 & 0 & 0 & 1 & 0 & 1 & 0 & 0 & 0 & 0 \\
0 & 0 & 0 & 0 & 1 & 0 & 1 & 1 & 0 & 0 \\
1 & 0 & 0 & 0 & 0 & 1 & 0 & 0 & 0 & 0 \\
1 & 0 & 0 & 0 & 0 & 1 & 0 & 0 & 0 & 0 \\
1 & 0 & 1 & 0 & 0 & 0 & 0 & 0 & 0 & 1 \\
0 & 0 & 0 & 1 & 0 & 0 & 0 & 0 & 1 & 0
\end{array}\right] \quad A M_{b}=\left[\begin{array}{llllllllll}
0 & 1 & 0 & 0 & 0 & 0 & 1 & 1 & 1 & 0 \\
1 & 0 & 1 & 0 & 0 & 0 & 0 & 0 & 0 & 1 \\
0 & 1 & 0 & 1 & 0 & 0 & 0 & 0 & 0 & 0 \\
0 & 0 & 1 & 0 & 1 & 0 & 0 & 0 & 0 & 1 \\
0 & 0 & 0 & 1 & 0 & 1 & 0 & 0 & 0 & 0 \\
0 & 0 & 0 & 0 & 1 & 0 & 1 & 1 & 0 & 0 \\
1 & 0 & 0 & 0 & 0 & 1 & 0 & 0 & 0 & 0 \\
1 & 0 & 0 & 0 & 0 & 1 & 0 & 0 & 0 & 0 \\
1 & 0 & 0 & 0 & 0 & 0 & 0 & 0 & 0 & 1 \\
0 & 1 & 0 & 1 & 0 & 0 & 0 & 0 & 1 & 0
\end{array}\right]
$$

Table 3. The PILs of the kinematic chains in Fig. 4

\begin{tabular}{ccccc}
\hline \multicolumn{3}{c}{ The kinematic chain in Fig. 5 (a) } & \multicolumn{2}{c}{ The kinematic chain in Fig. 5 (b) } \\
No. & \multicolumn{1}{c}{ PILs } & PILs in the form & PILs & PILs in the form \\
\multicolumn{7}{c}{ of the link degree } & & of the link degree \\
\hline 1 & $(1,2,3,4,5,6,7,1)$ & $(4,2,3,3,2,3,2,4)$ & $(1,2,3,4,5,6,7,1)$ & $(4,3,2,3,2,3,2,4)$ \\
2 & $(1,2,3,9,1)$ & $(4,2,3,3,4)$ & $(1,7,6,8,1)$ & $(4,2,3,2,4)$ \\
3 & $(1,7,6,8,1)$ & $(4,2,3,2,4)$ & $(1,2,10,9,1)$ & $(4,3,3,2,4)$ \\
4 & $(1,2,3,4,10,9,1)$ & $(4,2,3,3,2,3,4)$ & $(1,2,3,4,10,9,1)$ & $(4,3,2,3,3,2,4)$ \\
5 & $(1,7,6,5,4,10,9,1)$ & $(4,2,3,2,3,2,3,4)$ & $(1,2,10,4,5,6,7,1)$ & $(4,3,3,3,2,3,2,4)$ \\
6 & $(1,2,3,4,5,6,8,1)$ & $(4,2,3,3,2,3,2,4)$ & $(1,2,3,4,5,6,8,1)$ & $(4,3,2,3,2,3,2,4)$ \\
7 & $(1,7,6,5,4,3,9,1)$ & $(4,2,3,2,3,3,3,4)$ & $(1,2,10,4,5,6,8,1)$ & $(4,3,3,3,2,3,2,4)$ \\
8 & $(1,8,6,5,4,3,9,1)$ & $(4,2,3,2,3,3,3,4)$ & $(1,7,6,5,4,10,9,1)$ & $(4,2,3,2,3,3,2,4)$ \\
9 & $(1,8,6,5,4,10,9,1)$ & $(4,2,3,2,3,2,3,4)$ & $(1,8,6,5,4,10,9,1)$ & $(4,2,3,2,3,3,2,4)$ \\
10 & $(1,2,3,9,10,4,5,6$, & $(4,2,3,3,2,3,2,3$, & $(1,7,6,5,4,3,2,10$, & $(4,2,3,2,3,2,3,3$, \\
& $7,1)$ & $2,4)$ & $9,1)$ & $2,4)$ \\
11 & $(1,2,3,9,10,4,5,6$, & $(4,2,3,3,2,3,2,3$, & $(1,8,6,5,4,3,2,10$, & $(4,2,3,2,3,2,3,3$, \\
& $8,1)$ & $2,4)$ & $9,1)$ & $2,4)$
\end{tabular}

\subsection{Example}

Consider a set of kinematic chains with eleven links (Rai and Punjabi 2018). It is shown in Fig. 6.

The $A M s$ of kinematic chains in Fig. 6(a) and Fig. 6(b) are represented by $A M_{a}$ and $A M_{b}$ respectively.

After the procedures in section 3.2 are executed, the number of PILs in Fig. 6(a) is four. PILs include $(1,2,3,5,4,1),(1,7,8,9,10,1),(1,7,11,10,1)$ and $(1,2,3,6,4,1)$. PILs in the form of the link degree are $(4,2,3,2,3,4),(4,3,2,2,3,4),(4,3,2,3,4)$ and $(4,2,3,2,3,4)$.

In Fig. 6(b), the number of PILs is four. PILs include $(1,2,3,5,4,1),(1,8,7,10,9,1),(1,2,3,6,4$, 1), $(1,8,7,11,9,1)$. And PILs in the form of the link degree are $(4,2,3,2,3,4),(4,2,3,2,3,4),(4,2,3$, $2,3,4)$ and $(4,2,3,2,3,4)$. 
Based on the discriminant criterion, kinematic chains in Fig. 6 are not isomorphic.

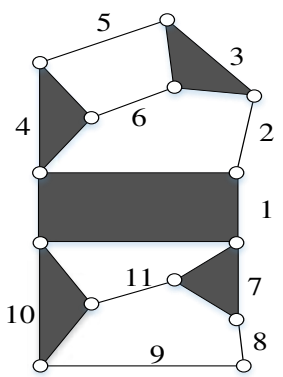

(a)

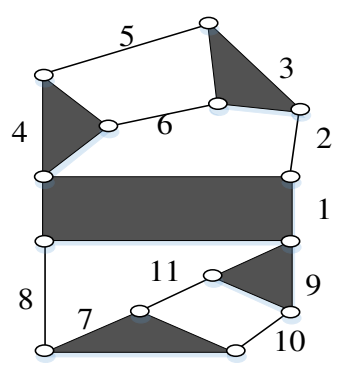

(b)

Fig. 6 The kinematic chain with eleven links

$$
A M_{a}=\left[\begin{array}{lllllllllll}
0 & 1 & 0 & 1 & 0 & 0 & 1 & 0 & 0 & 1 & 0 \\
1 & 0 & 1 & 0 & 0 & 0 & 0 & 0 & 0 & 0 & 0 \\
0 & 1 & 0 & 0 & 1 & 1 & 0 & 0 & 0 & 0 & 0 \\
1 & 0 & 0 & 0 & 1 & 1 & 0 & 0 & 0 & 0 & 0 \\
0 & 0 & 1 & 1 & 0 & 0 & 0 & 0 & 0 & 0 & 0 \\
0 & 0 & 1 & 1 & 0 & 0 & 0 & 0 & 0 & 0 & 0 \\
1 & 0 & 0 & 0 & 0 & 0 & 0 & 1 & 0 & 0 & 1 \\
0 & 0 & 0 & 0 & 0 & 0 & 1 & 0 & 1 & 0 & 0 \\
0 & 0 & 0 & 0 & 0 & 0 & 0 & 1 & 0 & 1 & 0 \\
1 & 0 & 0 & 0 & 0 & 0 & 0 & 0 & 1 & 0 & 1 \\
0 & 0 & 0 & 0 & 0 & 0 & 1 & 0 & 0 & 1 & 0
\end{array}\right] A M_{b}=\left[\begin{array}{lllllllllll}
0 & 1 & 0 & 1 & 0 & 0 & 0 & 1 & 1 & 0 & 0 \\
1 & 0 & 1 & 0 & 0 & 0 & 0 & 0 & 0 & 0 & 0 \\
0 & 1 & 0 & 0 & 1 & 1 & 0 & 0 & 0 & 0 & 0 \\
1 & 0 & 0 & 0 & 1 & 1 & 0 & 0 & 0 & 0 & 0 \\
0 & 0 & 1 & 1 & 0 & 0 & 0 & 0 & 0 & 0 & 0 \\
0 & 0 & 1 & 1 & 0 & 0 & 0 & 0 & 0 & 0 & 0 \\
0 & 0 & 0 & 0 & 0 & 0 & 0 & 1 & 0 & 1 & 1 \\
1 & 0 & 0 & 0 & 0 & 0 & 1 & 0 & 0 & 0 & 0 \\
1 & 0 & 0 & 0 & 0 & 0 & 0 & 0 & 0 & 1 & 1 \\
0 & 0 & 0 & 0 & 0 & 0 & 1 & 0 & 1 & 0 & 0 \\
0 & 0 & 0 & 0 & 0 & 0 & 1 & 0 & 1 & 0 & 0
\end{array}\right]
$$

\subsection{Example}

Consider a set of kinematic chains with twelve links (Madan 2014; Kamesh, Rao, and Rao 2017; Ding and Huang 2007). It is shown in Fig. 7.

The $A M s$ of kinematic chains in Fig. 7(a), Fig. 7(b), and Fig. 7(c) are represented by $A M_{a}, A M_{b}$, and $A M_{c}$ respectively.

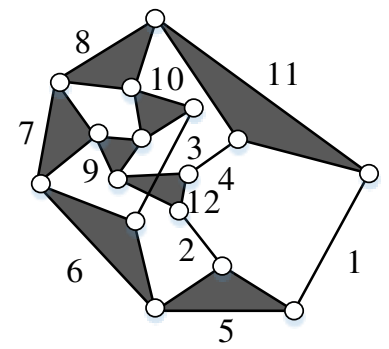

(a)

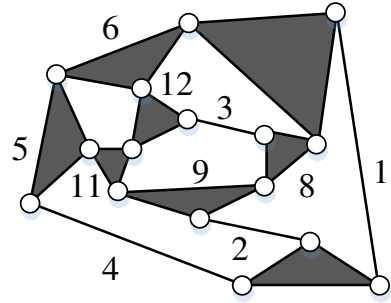

(b)

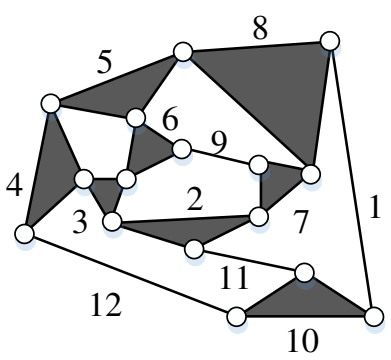

(c)

Fig. 7 The kinematic chain with twelve links 


$$
A M_{a}=\left[\begin{array}{llllllllllll}
0 & 0 & 0 & 0 & 1 & 0 & 0 & 0 & 0 & 0 & 1 & 0 \\
0 & 0 & 0 & 0 & 1 & 0 & 0 & 0 & 0 & 0 & 0 & 1 \\
0 & 0 & 0 & 0 & 0 & 1 & 0 & 0 & 0 & 1 & 0 & 0 \\
0 & 0 & 0 & 0 & 0 & 0 & 0 & 0 & 0 & 0 & 1 & 1 \\
1 & 1 & 0 & 0 & 0 & 1 & 0 & 0 & 0 & 0 & 0 & 0 \\
0 & 0 & 1 & 0 & 1 & 0 & 1 & 0 & 0 & 0 & 0 & 0 \\
0 & 0 & 0 & 0 & 0 & 1 & 0 & 1 & 1 & 0 & 0 & 0 \\
0 & 0 & 0 & 0 & 0 & 0 & 1 & 0 & 0 & 1 & 1 & 0 \\
0 & 0 & 0 & 0 & 0 & 0 & 1 & 0 & 0 & 1 & 0 & 1 \\
0 & 0 & 1 & 0 & 0 & 0 & 0 & 1 & 1 & 0 & 0 & 0 \\
1 & 0 & 0 & 1 & 0 & 0 & 0 & 1 & 0 & 0 & 0 & 0 \\
0 & 1 & 0 & 1 & 0 & 0 & 0 & 0 & 1 & 0 & 0 & 0
\end{array}\right] \quad A M_{b}=\left[\begin{array}{llllllllllll}
0 & 0 & 0 & 0 & 0 & 0 & 1 & 0 & 0 & 1 & 0 & 0 \\
0 & 0 & 0 & 0 & 0 & 0 & 0 & 0 & 1 & 1 & 0 & 0 \\
0 & 0 & 0 & 0 & 0 & 0 & 0 & 1 & 0 & 0 & 0 & 1 \\
0 & 0 & 0 & 0 & 1 & 0 & 0 & 0 & 0 & 1 & 0 & 0 \\
0 & 0 & 0 & 1 & 0 & 1 & 0 & 0 & 0 & 0 & 1 & 0 \\
0 & 0 & 0 & 0 & 1 & 0 & 1 & 0 & 0 & 0 & 0 & 1 \\
1 & 0 & 0 & 0 & 0 & 1 & 0 & 1 & 0 & 0 & 0 & 0 \\
0 & 0 & 1 & 0 & 0 & 0 & 1 & 0 & 1 & 0 & 0 & 0 \\
0 & 1 & 0 & 0 & 0 & 0 & 0 & 1 & 0 & 0 & 1 & 0 \\
1 & 1 & 0 & 1 & 0 & 0 & 0 & 0 & 0 & 0 & 0 & 0 \\
0 & 0 & 0 & 0 & 1 & 0 & 0 & 0 & 1 & 0 & 0 & 1 \\
0 & 0 & 1 & 0 & 0 & 1 & 0 & 0 & 0 & 0 & 1 & 0
\end{array}\right]
$$

$$
A M_{c}=\left[\begin{array}{llllllllllll}
0 & 0 & 0 & 0 & 0 & 0 & 0 & 1 & 0 & 1 & 0 & 0 \\
0 & 0 & 1 & 0 & 0 & 0 & 1 & 0 & 0 & 0 & 1 & 0 \\
0 & 1 & 0 & 1 & 0 & 1 & 0 & 0 & 0 & 0 & 0 & 0 \\
0 & 0 & 1 & 0 & 1 & 0 & 0 & 0 & 0 & 0 & 0 & 1 \\
0 & 0 & 0 & 1 & 0 & 1 & 0 & 1 & 0 & 0 & 0 & 0 \\
0 & 0 & 1 & 0 & 1 & 0 & 0 & 0 & 1 & 0 & 0 & 0 \\
0 & 1 & 0 & 0 & 0 & 0 & 0 & 1 & 1 & 0 & 0 & 0 \\
1 & 0 & 0 & 0 & 1 & 0 & 1 & 0 & 0 & 0 & 0 & 0 \\
0 & 0 & 0 & 0 & 0 & 1 & 1 & 0 & 0 & 0 & 0 & 0 \\
1 & 0 & 0 & 0 & 0 & 0 & 0 & 0 & 0 & 0 & 1 & 1 \\
0 & 1 & 0 & 0 & 0 & 0 & 0 & 0 & 0 & 1 & 0 & 0 \\
0 & 0 & 0 & 1 & 0 & 0 & 0 & 0 & 0 & 1 & 0 & 0
\end{array}\right]
$$

After the procedures in section 3.2 are executed, PILs in Fig. 7 are shown in Table 4, Table 5, and Table 6. Based on the discriminant criterion, the two kinematic chains in Fig. 7(a) and Fig. 7(b) are not isomorphic. However, the two kinematic chains in Fig. 7(b) and Fig. 7(c) are isomorphic.

Table 4. PILs of the kinematic chains in Fig. 7(a)

\begin{tabular}{lll}
\hline No. & \multicolumn{1}{c}{ PILs } & \multicolumn{1}{c}{ PILs in the form of the link degree } \\
\hline 1 & $(1,5,2,12,4,11,1)$ & $(2,3,2,3,2,3,2)$ \\
2 & $(1,5,6,3,10,9,7,8,11,1)$ & $(2,3,3,2,3,3,3,3,3,2)$ \\
3 & $(1,5,6,7,8,11,1)$ & $(2,3,3,3,3,3,2)$ \\
4 & $(1,5,2,12,9,10,3,6,7,8,11,1)$ & $(2,3,2,3,3,3,2,3,3,3,3,2)$ \\
5 & $(1,5,6,3,10,8,11,1)$ & $(2,3,3,2,3,3,3,2)$ \\
6 & $(1,5,2,12,9,7,6,3,10,8,11,1)$ & $(2,3,2,3,3,3,3,2,3,3,3,2)$ \\
7 & $(1,5,6,7,9,12,4,11,1)$ & $(2,3,3,3,3,3,2,3,2)$ \\
8 & $(1,5,6,7,9,10,8,11,1)$ & $(2,3,3,3,3,3,3,3,2)$ \\
9 & $(1,5,2,12,9,7,8,11,1)$ & $(2,3,2,3,3,3,3,3,2)$ \\
10 & $(1,5,6,3,10,9,12,4,11,1)$ & $(2,3,3,2,3,3,3,2,3,2)$ \\
11 & $(1,5,2,12,9,10,8,11,1)$ & $(2,3,2,3,3,3,3,3,2)$ \\
12 & $(1,5,6,3,10,8,7,9,12,4,11,1)$ & $(2,3,3,2,3,3,3,3,3,2,3,2)$ \\
13 & $(1,5,6,7,8,10,9,12,4,11,1)$ & $(2,3,3,3,3,3,3,3,2,3,2)$ \\
\hline
\end{tabular}


Table 5. PILs of the kinematic chains in Fig. 7(b)

\begin{tabular}{cll}
\hline No. & \multicolumn{1}{c}{ PILs } & \multicolumn{1}{c}{ PILs in the form of the link degree } \\
\hline 1 & $(1,7,6,5,4,10,1)$ & $(2,3,3,3,2,3,2)$ \\
2 & $(1,7,8,9,2,10,1)$ & $(2,3,3,3,2,3,2)$ \\
3 & $(1,7,8,3,12,6,5,4,10,1)$ & $(2,3,3,2,3,3,3,2,3,2)$ \\
4 & $(1,7,8,3,12,11,5,4,10,1)$ & $(2,3,3,2,3,3,3,2,3,2)$ \\
5 & $(1,7,6,5,11,9,2,10,1)$ & $(2,3,3,3,3,3,2,3,2)$ \\
6 & $(1,7,8,3,12,11,9,2,10,1)$ & $(2,3,3,2,3,3,3,2,3,2)$ \\
7 & $(1,7,6,12,11,5,4,10,1)$ & $(2,3,3,3,3,3,2,3,2)$ \\
8 & $(1,7,6,12,3,8,9,11,5,4,10,1)$ & $(2,3,3,3,2,3,3,3,3,2,3,2)$ \\
9 & $(1,7,8,3,12,6,5,11,9,2,10,1)$ & $(2,3,3,2,3,3,3,3,3,2,3,2)$ \\
10 & $(1,7,8,9,11,5,4,10,1)$ & $(2,3,3,3,3,3,2,3,2)$ \\
11 & $(1,7,6,12,11,9,2,10,1)$ & $(2,3,3,3,3,3,2,3,2)$ \\
12 & $(1,7,6,12,3,8,9,2,10,1)$ & $(2,3,3,3,2,3,3,2,3,2)$ \\
13 & $(1,7,6,5,11,12,3,8,9,2,10,1)$ & $(2,3,3,3,3,3,2,3,3,2,3,2)$ \\
14 & $(1,7,8,9,11,12,6,5,4,10,1)$ & $(2,3,3,3,3,3,3,3,2,3,2)$ \\
\hline
\end{tabular}

Table 6. PILs of the kinematic chains in Fig. 7(c)

\begin{tabular}{cll}
\hline No. & \multicolumn{1}{c}{ PILs } & \multicolumn{1}{c}{ PILs in the form of the link degree } \\
\hline 1 & $(1,8,7,2,11,10,1)$ & $(2,3,3,3,2,3,2)$ \\
2 & $(1,8,7,2,3,6,5,4,12,10,1)$ & $(2,3,3,3,3,3,3,3,2,3,2)$ \\
3 & $(1,8,5,6,3,4,12,10,1)$ & $(2,3,3,3,3,3,2,3,2)$ \\
4 & $(1,8,7,9,6,5,4,3,2,11,10,1)$ & $(2,3,3,2,3,3,3,3,3,2,3,2)$ \\
5 & $(1,8,5,4,3,6,9,7,2,11,10,1)$ & $(2,3,3,3,3,3,2,3,3,2,3,2)$ \\
6 & $(1,8,5,4,12,10,1)$ & $(2,3,3,3,2,3,2)$ \\
7 & $(1,8,7,2,3,4,12,10,1)$ & $(2,3,3,3,3,3,2,3,2)$ \\
8 & $(1,8,7,9,6,3,2,11,10,1)$ & $(2,3,3,2,3,3,3,2,3,2)$ \\
9 & $(1,8,5,4,3,2,11,10,1)$ & $(2,3,3,3,3,3,2,3,2)$ \\
10 & $(1,8,5,6,9,7,2,3,4,12,10,1)$ & $(2,3,3,3,2,3,3,3,3,2,3,2)$ \\
11 & $(1,8,5,6,3,2,11,10,1)$ & $(2,3,3,3,3,3,2,3,2)$ \\
12 & $(1,8,7,9,6,5,4,12,10,1)$ & $(2,3,3,2,3,3,3,2,3,2)$ \\
13 & $(1,8,7,9,6,3,4,12,10,1)$ & $(2,3,3,2,3,3,3,2,3,2)$ \\
14 & $(1,8,5,6,9,7,2,11,10,1)$ & $(2,3,3,3,2,3,3,2,3,2)$ \\
\hline
\end{tabular}

\subsection{Example}

For the topology graph, vertices of the graph denote the links of the kinematic chain, and edges of the graph denote the joints of the kinematic chain. Consider a set of topology graphs with fifteen vertices (He, Zhang, and Li 2005; Ding and Huang 2007; Gloria, Enrique, and Domingo 2007). It is shown in Fig. 9.

The $A M s$ of topology graphs in Fig. 8(a) and Fig. 8(b) are represented by $A M_{a}$ and $A M_{b}$ respectively. 


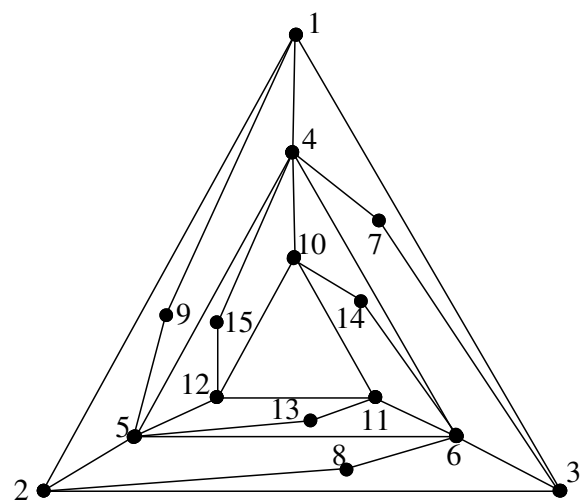

(a)

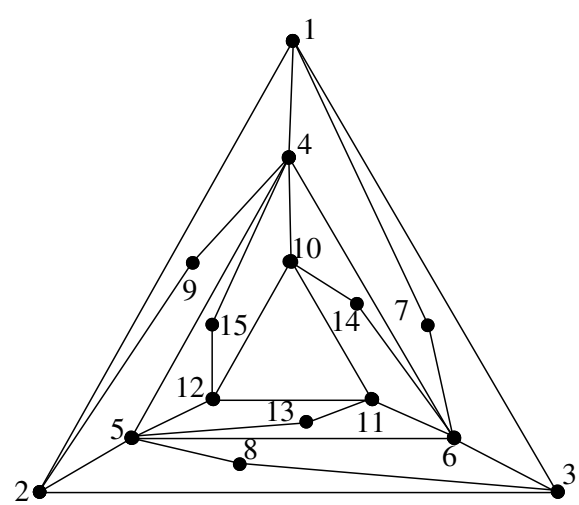

(b)

Fig. 8 The topology graphs with fifteen vertices

$$
\begin{aligned}
& A M_{a}=\left[\begin{array}{lllllllllllllll}
0 & 1 & 1 & 1 & 0 & 0 & 0 & 0 & 1 & 0 & 0 & 0 & 0 & 0 & 0 \\
1 & 0 & 1 & 0 & 1 & 0 & 0 & 1 & 0 & 0 & 0 & 0 & 0 & 0 & 0 \\
1 & 1 & 0 & 0 & 0 & 1 & 1 & 0 & 0 & 0 & 0 & 0 & 0 & 0 & 0 \\
1 & 0 & 0 & 0 & 1 & 1 & 1 & 0 & 0 & 1 & 0 & 0 & 0 & 0 & 1 \\
0 & 1 & 0 & 1 & 0 & 1 & 0 & 0 & 1 & 0 & 0 & 1 & 1 & 0 & 0 \\
0 & 0 & 1 & 1 & 1 & 0 & 0 & 1 & 0 & 0 & 1 & 0 & 0 & 1 & 0 \\
0 & 0 & 1 & 1 & 0 & 0 & 0 & 0 & 0 & 0 & 0 & 0 & 0 & 0 & 0 \\
0 & 1 & 0 & 0 & 0 & 1 & 0 & 0 & 0 & 0 & 0 & 0 & 0 & 0 & 0 \\
1 & 0 & 0 & 0 & 1 & 0 & 0 & 0 & 0 & 0 & 0 & 0 & 0 & 0 & 0 \\
0 & 0 & 0 & 1 & 0 & 0 & 0 & 0 & 0 & 0 & 1 & 1 & 0 & 1 & 0 \\
0 & 0 & 0 & 0 & 0 & 1 & 0 & 0 & 0 & 1 & 0 & 1 & 1 & 0 & 0 \\
0 & 0 & 0 & 0 & 1 & 0 & 0 & 0 & 0 & 1 & 1 & 0 & 0 & 0 & 1 \\
0 & 0 & 0 & 0 & 1 & 0 & 0 & 0 & 0 & 0 & 1 & 0 & 0 & 0 & 0 \\
0 & 0 & 0 & 0 & 0 & 1 & 0 & 0 & 0 & 1 & 0 & 0 & 0 & 0 & 0 \\
0 & 0 & 0 & 1 & 0 & 0 & 0 & 0 & 0 & 0 & 0 & 1 & 0 & 0 & 0
\end{array}\right] \\
& A M_{b}=\left[\begin{array}{lllllllllllllll}
0 & 1 & 1 & 1 & 0 & 0 & 1 & 0 & 0 & 0 & 0 & 0 & 0 & 0 & 0 \\
1 & 0 & 1 & 0 & 1 & 0 & 0 & 0 & 1 & 0 & 0 & 0 & 0 & 0 & 0 \\
1 & 1 & 0 & 0 & 0 & 1 & 0 & 1 & 0 & 0 & 0 & 0 & 0 & 0 & 0 \\
1 & 0 & 0 & 0 & 1 & 1 & 0 & 0 & 1 & 1 & 0 & 0 & 0 & 0 & 1 \\
0 & 1 & 0 & 1 & 0 & 1 & 0 & 1 & 0 & 0 & 0 & 1 & 1 & 0 & 0 \\
0 & 0 & 1 & 1 & 1 & 0 & 1 & 0 & 0 & 0 & 1 & 0 & 0 & 1 & 0 \\
1 & 0 & 0 & 0 & 0 & 1 & 0 & 0 & 0 & 0 & 0 & 0 & 0 & 0 & 0 \\
0 & 0 & 1 & 0 & 1 & 0 & 0 & 0 & 0 & 0 & 0 & 0 & 0 & 0 & 0 \\
0 & 1 & 0 & 1 & 0 & 0 & 0 & 0 & 0 & 0 & 0 & 0 & 0 & 0 & 0 \\
0 & 0 & 0 & 1 & 0 & 0 & 0 & 0 & 0 & 0 & 1 & 1 & 0 & 1 & 0 \\
0 & 0 & 0 & 0 & 0 & 1 & 0 & 0 & 0 & 1 & 0 & 1 & 1 & 0 & 0 \\
0 & 0 & 0 & 0 & 1 & 0 & 0 & 0 & 0 & 1 & 1 & 0 & 0 & 0 & 1 \\
0 & 0 & 0 & 0 & 1 & 0 & 0 & 0 & 0 & 0 & 1 & 0 & 0 & 0 & 0 \\
0 & 0 & 0 & 0 & 0 & 1 & 0 & 0 & 0 & 1 & 0 & 0 & 0 & 0 & 0 \\
0 & 0 & 0 & 1 & 0 & 0 & 0 & 0 & 0 & 0 & 0 & 1 & 0 & 0 & 0
\end{array}\right]
\end{aligned}
$$




\begin{tabular}{|c|c|c|c|c|c|c|c|c|c|c|c|c|c|c|c|c|}
\hline \multicolumn{10}{|l}{ 490x 16 double } \\
\hline & 1 & 2 & 3 & 4 & 5 & 6 & 7 & 8 & 9 & 10 & 11 & 12 & 13 & 14 & 15 & 16 \\
\hline 1 & 4 & 4 & 4 & 4 & 0 & 0 & 0 & 0 & 0 & 0 & 0 & 0 & 0 & 0 & 0 & 0 \\
\hline 2 & 4 & 4 & 6 & 6 & 4 & 0 & 0 & 0 & 0 & 0 & 0 & 0 & 0 & 0 & 0 & 0 \\
\hline 3 & 4 & 4 & 6 & 6 & 4 & 0 & 0 & 0 & 0 & 0 & 0 & 0 & 0 & 0 & 0 & 0 \\
\hline 4 & 4 & 4 & 2 & 6 & 4 & 0 & 0 & 0 & 0 & 0 & 0 & 0 & 0 & 0 & 0 & 0 \\
\hline 5 & 4 & 6 & 6 & 2 & 4 & 0 & 0 & 0 & 0 & 0 & 0 & 0 & 0 & 0 & 0 & 0 \\
\hline 6 & 4 & 4 & 6 & 2 & 4 & 0 & 0 & 0 & 0 & 0 & 0 & 0 & 0 & 0 & 0 & 0 \\
\hline 7 & 4 & 4 & 6 & 6 & 4 & 4 & 0 & 0 & 0 & 0 & 0 & 0 & 0 & 0 & 0 & 0 \\
\hline 8 & 4 & 4 & 2 & 6 & 4 & 4 & 0 & 0 & 0 & 0 & 0 & 0 & 0 & 0 & 0 & 0 \\
\hline 9 & 4 & 4 & 6 & 4 & 4 & 6 & 4 & 0 & 0 & 0 & 0 & 0 & 0 & 0 & 0 & 0 \\
\hline 10 & 4 & 4 & 6 & 4 & 4 & 6 & 4 & 0 & 0 & 0 & 0 & 0 & 0 & 0 & 0 & 0 \\
\hline 11 & 4 & 4 & 6 & 2 & 4 & 6 & 4 & 0 & 0 & 0 & 0 & 0 & 0 & 0 & 0 & 0 \\
\hline 12 & 4 & 4 & 4 & 6 & 6 & 4 & 0 & 0 & 0 & 0 & 0 & 0 & 0 & 0 & 0 & 0 \\
\hline 13 & 4 & 4 & 2 & 6 & 6 & 4 & 0 & 0 & 0 & 0 & 0 & 0 & 0 & 0 & 0 & 0 \\
\hline 14 & 4 & 4 & 6 & 6 & 6 & 4 & 4 & 0 & 0 & 0 & 0 & 0 & 0 & 0 & 0 & 0 \\
\hline 15 & 4 & 4 & 6 & 6 & 2 & 4 & 4 & 0 & 0 & 0 & 0 & 0 & 0 & 0 & 0 & 0 \\
\hline 16 & 4 & 4 & 4 & 6 & 6 & 4 & 0 & 0 & 0 & 0 & 0 & 0 & 0 & 0 & 0 & 0 \\
\hline
\end{tabular}

(a) Excerpt of the 490 PILs in the form of link degree in Fig. 8(a)

\begin{tabular}{|c|c|c|c|c|c|c|c|c|c|c|c|c|c|c|c|c|}
\hline 四 & 490x16 double \\
\hline & 1 & 2 & 3 & 4 & 5 & 6 & 7 & 8 & 9 & 10 & 11 & 12 & 13 & 14 & 15 & 16 \\
\hline 1 & 4 & 4 & 4 & 4 & 0 & 0 & 0 & 0 & 0 & 0 & 0 & 0 & 0 & 0 & 0 & 0 \\
\hline 2 & 4 & 4 & 6 & 6 & 4 & 0 & 0 & 0 & 0 & 0 & 0 & 0 & 0 & 0 & 0 & 0 \\
\hline 3 & 4 & 4 & 6 & 6 & 4 & 0 & 0 & 0 & 0 & 0 & 0 & 0 & 0 & 0 & 0 & 0 \\
\hline 4 & 4 & 4 & 2 & 6 & 4 & 0 & 0 & 0 & 0 & 0 & 0 & 0 & 0 & 0 & 0 & 0 \\
\hline 5 & 4 & 4 & 6 & 2 & 4 & 4 & 0 & 0 & 0 & 0 & 0 & 0 & 0 & 0 & 0 & 0 \\
\hline 6 & 4 & 4 & 6 & 6 & 4 & 4 & 0 & 0 & 0 & 0 & 0 & 0 & 0 & 0 & 0 & 0 \\
\hline 7 & 4 & 4 & 6 & 2 & 4 & 0 & 0 & 0 & 0 & 0 & 0 & 0 & 0 & 0 & 0 & 0 \\
\hline 8 & 4 & 4 & 6 & 4 & 4 & 6 & 4 & 0 & 0 & 0 & 0 & 0 & 0 & 0 & 0 & 0 \\
\hline 9 & 4 & 6 & 6 & 2 & 4 & 0 & 0 & 0 & 0 & 0 & 0 & 0 & 0 & 0 & 0 & 0 \\
\hline 10 & 4 & 4 & 6 & 4 & 4 & 6 & 4 & 0 & 0 & 0 & 0 & 0 & 0 & 0 & 0 & 0 \\
\hline 11 & 4 & 4 & 6 & 2 & 4 & 6 & 4 & 0 & 0 & 0 & 0 & 0 & 0 & 0 & 0 & 0 \\
\hline 12 & 4 & 4 & 4 & 6 & 6 & 4 & 0 & 0 & 0 & 0 & 0 & 0 & 0 & 0 & 0 & 0 \\
\hline 13 & 4 & 4 & 6 & 6 & 6 & 4 & 4 & 0 & 0 & 0 & 0 & 0 & 0 & 0 & 0 & 0 \\
\hline 14 & 4 & 4 & 2 & 6 & 6 & 4 & 4 & 0 & 0 & 0 & 0 & 0 & 0 & 0 & 0 & 0 \\
\hline 15 & 4 & 4 & 2 & 6 & 6 & 4 & 0 & 0 & 0 & 0 & 0 & 0 & 0 & 0 & 0 & 0 \\
\hline 16 & 4 & 4 & 4 & 6 & 6 & 4 & 0 & 0 & 0 & 0 & 0 & 0 & 0 & 0 & 0 & 0 \\
\hline
\end{tabular}

(b) Excerpt of the 490 PILs in the form of link degree in Fig. 8(b)

Fig. 9 Excerpt of the 490 PILs in the form of link degree in Fig. 8

The number of PILs in Fig. 8(a) and Fig. 8(b) are both 490. However, the two topology graphs in Fig. 8 are not isomorphic because PILs in the form of the link degree are not one-to-one correspondences.

\subsection{Example}

Consider a set of kinematic chains with multiple joints (Zou and He 2016). It is shown in Fig. 10.

The $A M s$ of kinematic chains in Fig. 10(a) and Fig. 10(b) are represented by $A M_{a}$ and $A M_{b}$ respectively. 


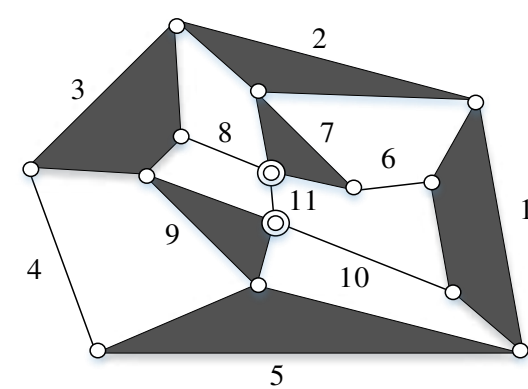

(a)

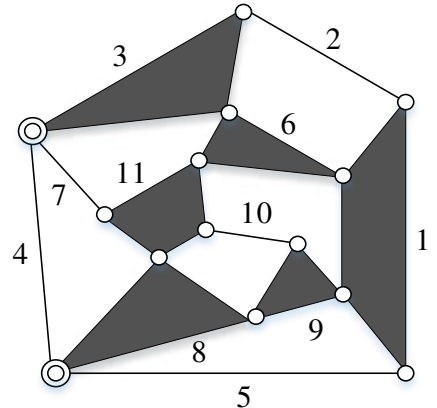

(b)

Fig. 10 Two11-link kinematic chains with multiple joints

\begin{tabular}{|c|c|c|c|c|c|c|c|c|c|c|c|c|c|c|c|c|c|c|c|c|c|c|c|c|c|}
\hline \multicolumn{13}{|c|}{$\boxplus 36 \times 12$ double } & \multicolumn{13}{|c|}{$\boxplus 36 \times 12$ double } \\
\hline & 1 & 2 & 3 & 4 & 5 & 6 & 7 & 8 & 9 & 10 & 11 & 12 & & 1 & 2 & 3 & 4 & 5 & 6 & 7 & 8 & 9 & 10 & 11 & 12 \\
\hline 1 & 4 & 3 & 4 & 2 & 3 & 4 & 0 & 0 & 0 & 0 & 0 & 0 & 1 & 4 & 2 & 4 & 4 & 3 & 4 & 0 & 0 & 0 & 0 & 0 & 0 \\
\hline 2 & 4 & 3 & 4 & 2 & 4 & 0 & 0 & 0 & 0 & 0 & 0 & 0 & 2 & 4 & 2 & 4 & 3 & 4 & 0 & 0 & 0 & 0 & 0 & 0 & 0 \\
\hline 3 & 4 & 3 & 4 & 4 & 3 & 4 & 0 & 0 & 0 & 0 & 0 & 0 & 3 & 4 & 3 & 4 & 3 & 4 & 0 & 0 & 0 & 0 & 0 & 0 & 0 \\
\hline 4 & 4 & 3 & 4 & 3 & 4 & 2 & 4 & 0 & 0 & 0 & 0 & 0 & 4 & 4 & 3 & 4 & 2 & 3 & 4 & 0 & 0 & 0 & 0 & 0 & 0 \\
\hline 5 & 4 & 3 & 4 & 3 & 4 & 0 & 0 & 0 & 0 & 0 & 0 & 0 & 5 & 4 & 3 & 4 & 4 & 3 & 4 & 0 & 0 & 0 & 0 & 0 & 0 \\
\hline 6 & 4 & 3 & 4 & 3 & 4 & 2 & 3 & 4 & 0 & 0 & 0 & 0 & 6 & 4 & 2 & 4 & 3 & 4 & 3 & 4 & 0 & 0 & 0 & 0 & 0 \\
\hline 7 & 4 & 3 & 2 & 4 & 4 & 3 & 4 & 0 & 0 & 0 & 0 & 0 & 7 & 4 & 3 & 4 & 4 & 3 & 4 & 0 & 0 & 0 & 0 & 0 & 0 \\
\hline 8 & 4 & 3 & 4 & 4 & 3 & 4 & 0 & 0 & 0 & 0 & 0 & 0 & 8 & 4 & 3 & 4 & 4 & 3 & 4 & 0 & 0 & 0 & 0 & 0 & 0 \\
\hline 9 & 4 & 3 & 4 & 4 & 4 & 4 & 2 & 4 & 0 & 0 & 0 & 0 & 9 & 4 & 2 & 4 & 4 & 4 & 3 & 4 & 0 & 0 & 0 & 0 & 0 \\
\hline 10 & 4 & 2 & 4 & 4 & 3 & 4 & 0 & 0 & 0 & 0 & 0 & 0 & 10 & 4 & 2 & 4 & 4 & 4 & 4 & 3 & 4 & 0 & 0 & 0 & 0 \\
\hline 11 & 4 & 3 & 4 & 4 & 3 & 4 & 0 & 0 & 0 & 0 & 0 & 0 & 11 & 4 & 3 & 4 & 3 & 4 & 3 & 4 & 0 & 0 & 0 & 0 & 0 \\
\hline 12 & 4 & 3 & 4 & 2 & 3 & 4 & 3 & 4 & 0 & 0 & 0 & 0 & 12 & 4 & 3 & 4 & 4 & 2 & 3 & 4 & 0 & 0 & 0 & 0 & 0 \\
\hline 13 & 4 & 3 & 2 & 4 & 3 & 4 & 2 & 4 & 0 & 0 & 0 & 0 & 13 & 4 & 2 & 4 & 3 & 4 & 2 & 3 & 4 & 0 & 0 & 0 & 0 \\
\hline 14 & 4 & 3 & 4 & 3 & 4 & 4 & 3 & 4 & 0 & 0 & 0 & 0 & 14 & 4 & 2 & 4 & 3 & 4 & 2 & 3 & 4 & 0 & 0 & 0 & 0 \\
\hline 15 & 4 & 3 & 4 & 4 & 4 & 4 & 2 & 3 & 4 & 0 & 0 & 0 & 15 & 4 & 3 & 4 & 4 & 4 & 3 & 4 & 0 & 0 & 0 & 0 & 0 \\
\hline 16 & 4 & 3 & 4 & 4 & 3 & 4 & 2 & 4 & 0 & 0 & 0 & 0 & 16 & 4 & 3 & 4 & 3 & 4 & 2 & 3 & 4 & 0 & 0 & 0 & 0 \\
\hline 17 & 4 & 3 & 4 & 3 & 4 & 4 & 3 & 4 & 0 & 0 & 0 & 0 & 17 & 4 & 2 & 4 & 3 & 4 & 3 & 4 & 3 & 4 & 0 & 0 & 0 \\
\hline 18 & 4 & 3 & 4 & 3 & 4 & 3 & 4 & 0 & 0 & 0 & 0 & 0 & 18 & 4 & 3 & 4 & 3 & 4 & 4 & 3 & 4 & 0 & 0 & 0 & 0 \\
\hline 19 & 4 & 3 & 4 & 4 & 4 & 2 & 4 & 0 & 0 & 0 & 0 & 0 & 19 & 4 & 3 & 4 & 4 & 3 & 4 & 3 & 4 & 0 & 0 & 0 & 0 \\
\hline 20 & 4 & 3 & 4 & 4 & 4 & 3 & 4 & 0 & 0 & 0 & 0 & 0 & 20 & 4 & 3 & 4 & 3 & 2 & 4 & 3 & 4 & 0 & 0 & 0 & 0 \\
\hline 21 & 4 & 3 & 2 & 4 & 3 & 4 & 2 & 4 & 0 & 0 & 0 & 0 & 21 & 4 & 2 & 4 & 4 & 4 & 3 & 2 & 4 & 3 & 4 & 0 & 0 \\
\hline 22 & 4 & 3 & 4 & 4 & 3 & 4 & 2 & 4 & 0 & 0 & 0 & 0 & 22 & 4 & 2 & 4 & 4 & 4 & 4 & 2 & 3 & 4 & 0 & 0 & 0 \\
\hline 23 & 4 & 2 & 4 & 3 & 4 & 4 & 3 & 4 & 0 & 0 & 0 & 0 & 23 & 4 & 3 & 4 & 4 & 3 & 4 & 4 & 3 & 4 & 0 & 0 & 0 \\
\hline 24 & 4 & 3 & 4 & 3 & 4 & 4 & 3 & 4 & 0 & 0 & 0 & 0 & 24 & 4 & 3 & 4 & 4 & 3 & 4 & 2 & 3 & 4 & 0 & 0 & 0 \\
\hline 25 & 4 & 3 & 2 & 4 & 3 & 4 & 3 & 4 & 0 & 0 & 0 & 0 & 25 & 4 & 3 & 4 & 3 & 4 & 4 & 3 & 4 & 0 & 0 & 0 & 0 \\
\hline 26 & 4 & 2 & 4 & 3 & 4 & 4 & 3 & 4 & 0 & 0 & 0 & 0 & 26 & 4 & 3 & 4 & 3 & 4 & 2 & 3 & 4 & 0 & 0 & 0 & 0 \\
\hline 27 & 4 & 3 & 4 & 4 & 3 & 4 & 3 & 4 & 0 & 0 & 0 & 0 & 27 & 4 & 2 & 4 & 3 & 4 & 4 & 3 & 4 & 0 & 0 & 0 & 0 \\
\hline 28 & 4 & 3 & 4 & 2 & 3 & 4 & 4 & 4 & 2 & 4 & 0 & 0 & 28 & 4 & 2 & 4 & 3 & 4 & 4 & 3 & 4 & 0 & 0 & 0 & 0 \\
\hline 29 & 4 & 3 & 4 & 3 & 4 & 2 & 3 & 4 & 3 & 4 & 0 & 0 & 29 & 4 & 2 & 4 & 3 & 4 & 4 & 3 & 4 & 0 & 0 & 0 & 0 \\
\hline 30 & 4 & 2 & 4 & 3 & 4 & 2 & 3 & 4 & 3 & 4 & 0 & 0 & 30 & 4 & 2 & 4 & 3 & 4 & 4 & 3 & 4 & 0 & 0 & 0 & 0 \\
\hline 31 & 4 & 3 & 4 & 4 & 3 & 4 & 3 & 4 & 2 & 4 & 0 & 0 & 31 & 4 & 3 & 4 & 3 & 4 & 4 & 3 & 4 & 0 & 0 & 0 & 0 \\
\hline 32 & 4 & 3 & 2 & 4 & 4 & 4 & 4 & 2 & 4 & 0 & 0 & 0 & 32 & 4 & 3 & 4 & 4 & 4 & 4 & 2 & 3 & 4 & 0 & 0 & 0 \\
\hline 33 & 4 & 2 & 4 & 3 & 4 & 3 & 4 & 3 & 4 & 0 & 0 & 0 & 33 & 4 & 2 & 4 & 3 & 4 & 3 & 4 & 4 & 3 & 4 & 0 & 0 \\
\hline 34 & 4 & 3 & 2 & 4 & 3 & 4 & 4 & 3 & 4 & 0 & 0 & 0 & 34 & 4 & 2 & 4 & 3 & 4 & 2 & 3 & 4 & 3 & 4 & 0 & 0 \\
\hline 35 & 4 & 3 & 4 & 4 & 3 & 4 & 4 & 3 & 4 & 0 & 0 & 0 & 35 & 4 & 2 & 4 & 3 & 4 & 2 & 3 & 4 & 3 & 4 & 0 & 0 \\
\hline 36 & 4 & 2 & 4 & 3 & 4 & 2 & 3 & 4 & 3 & 4 & 0 & 0 & 36 & 4 & 3 & 4 & 3 & 2 & 4 & 3 & 4 & 3 & 4 & 0 & 0 \\
\hline
\end{tabular}

(a) PILs in the form of link degree in Fig. 10(a) (b) PILs in the form of link degree in Fig. 10(b)

Fig. 11 PILs in the form of link degree in Fig. 10 


$$
A M_{a}=\left[\begin{array}{lllllllllll}
0 & 1 & 0 & 0 & 1 & 1 & 0 & 0 & 0 & 1 & 0 \\
1 & 0 & 1 & 0 & 0 & 0 & 1 & 0 & 0 & 0 & 0 \\
0 & 1 & 0 & 1 & 0 & 0 & 0 & 1 & 1 & 0 & 0 \\
0 & 0 & 1 & 0 & 1 & 0 & 0 & 0 & 0 & 0 & 0 \\
1 & 0 & 0 & 1 & 0 & 0 & 0 & 0 & 1 & 0 & 0 \\
1 & 0 & 0 & 0 & 0 & 0 & 1 & 0 & 0 & 0 & 0 \\
0 & 1 & 0 & 0 & 0 & 1 & 0 & 1 & 0 & 0 & 1 \\
0 & 0 & 1 & 0 & 0 & 0 & 1 & 0 & 0 & 0 & 1 \\
0 & 0 & 1 & 0 & 1 & 0 & 0 & 0 & 0 & 1 & 1 \\
1 & 0 & 0 & 0 & 0 & 0 & 0 & 0 & 1 & 0 & 1 \\
0 & 0 & 0 & 0 & 0 & 0 & 1 & 1 & 1 & 1 & 0
\end{array}\right] A M_{b}=\left[\begin{array}{lllllllllll}
0 & 1 & 0 & 0 & 1 & 1 & 0 & 0 & 1 & 0 & 0 \\
1 & 0 & 1 & 0 & 0 & 0 & 0 & 0 & 0 & 0 & 0 \\
0 & 1 & 0 & 1 & 0 & 1 & 1 & 0 & 0 & 0 & 0 \\
0 & 0 & 1 & 0 & 1 & 0 & 1 & 1 & 0 & 0 & 0 \\
1 & 0 & 0 & 1 & 0 & 0 & 0 & 1 & 0 & 0 & 0 \\
1 & 0 & 1 & 0 & 0 & 0 & 0 & 0 & 0 & 0 & 1 \\
0 & 0 & 1 & 1 & 0 & 0 & 0 & 0 & 0 & 0 & 1 \\
0 & 0 & 0 & 1 & 1 & 0 & 0 & 0 & 1 & 0 & 1 \\
1 & 0 & 0 & 0 & 0 & 0 & 0 & 1 & 0 & 1 & 0 \\
0 & 0 & 0 & 0 & 0 & 0 & 0 & 0 & 1 & 0 & 1 \\
0 & 0 & 0 & 0 & 0 & 1 & 1 & 1 & 0 & 1 & 0
\end{array}\right]
$$

The number of PILs in Fig. 10(a) and Fig. 10(b) are both 36. Two kinematic chains in Fig. 10 are isomorphic because PILs in the form of the link degree in Fig. 11 are one-to-one correspondences.

\section{Discussion}

The proposed method is very easy to be implemented, although it seems more complex at first sight. It's necessary to verify the correctness of the proposed method by comparing it with previous methods mentioned in the references.

(1) Kinematic chains in Fig. 1, Fig. 4, and Fig. 5 are isomorphic respectively. Because PILs in the form of the link degree in the kinematic chains are one-to-one correspondences. Results of isomorphism identification in Fig. 1 are consistent with that by visual inspection. The result of isomorphism identification in Fig. 4 is consistent with that by the kinematic chain label (Madan 2014) and graph theory (Kamesh, Rao, and Rao 2017). The result of isomorphism identification in Fig. 5 is consistent with that by graph theory (Kamesh, Rao, and Rao 2017) and a method based on the flow path matrices of kinematic chains (Dargar, Hasan, and Khan 2011).

(2) For 11-link kinematic chains, PILs in the form of the link degree in Fig. 6(a) and Fig. 6(b) are not one-to-one correspondences. Two kinematic chains are not isomorphic. The result is consistent with that by binary code (Rai and Punjabi 2018). For the fractionated kinematic chains, it's essential to select link 1 as the home link in Fig. 6. Otherwise, the result of isomorphism identification is not convincing. For example, if link 3 is selected as the home link, the loop will not contain link 7 to link 11. Under this circumstance, the result is wrong. Therefore, it's important to select the home link for fractioned kinematic chains.

(3) For 12-link kinematic chains, the number of PILs in Fig. 7(a) is less than that in Fig. 7(b) or Fig. 7(c). PILs in the form of the link degree in Fig. 7(b) and Fig. 7(c) are one-to-one correspondences. So kinematic chains in Fig. 7(a) and Fig. 7(b) or Fig. 7(c) are not isomorphic. Kinematic chains in Fig. 7(b) and Fig. 7(c) are isomorphic but labeled differently. Results are consistent with that by kinematic chain label (Madan 2014), graph theory (Kamesh, Rao, and Rao 2017) and the canonical perimeter topological graph (Ding and Huang 2007). To some extent, the example confirms that the labeled numbers of links don't alter the results of isomorphism identification.

(4) The number of PILs in topology graphs with fifteen vertices in Fig. 8(a) and Fig. 8(b) are equal. However, PILs in the form of the link degree are not one-to-one correspondences. They are not isomorphic. 
The result is consistent with that by the eigensystem approach (He, Zhang, and Li 2005), the canonical perimeter topological graph (Ding and Huang 2007), and improving neural networks (Gloria, Enrique, and Domingo 2007). Besides, the example illustrates that the proposed method is also suitable for isomorphism identification in the topology graphs.

(5) There are two 11-link kinematic chains with multiple joints in Fig. 9. PILs in the form of link degree in Fig. 9(a) and Fig. 9(b) are one-to-one correspondences. Kinematic chains in Fig. 9 are isomorphic. The result is consistent with that by the algorithm based on the weighted-double-colour-contracted-graph (Zou and He 2016). Therefore, the proposed method in this paper is also applicable to kinematic chains with multiple joints.

\section{Conclusions}

This paper proposes a new method to solve the isomorphism problem in the kinematic chains. The proposed method is very simple and efficient. It can be easily implemented on the computer. A program is written in MATLAB to obtain all PILs and justify whether kinematic chains are isomorphic. Compared with previous methods, the proposed method has been proven very effective and efficient in isomorphism identification. And it applies to kinematic chains and topological graphs.

The proposed method focuses on the basic features of kinematic chains, such as link assortment and the relative relation among links and vertices, to establish a connection from PIL to isomorphism identification. It relies on the selection of the home link, rather than the direction and sequence of labeled links. It can be considered the general method for isomorphism identification in the planar kinematic chains.

\section{Acknowledgments}

The authors would like to thank the Research and Development Plan of Shandong Province, China (Grant No. 2017CXGC0909) and the Intelligent Manufacturing Integrated Standardization and New Model Application Project (Grant No. 2016-213-3), respectively, for the financial support of the work.

\section{Compliance with ethical standards}

Conflict of interest The authors declare that they have no conflict of interest. All authors contributed to the study conception and design. All authors read and approved the final manuscript.

Ethical approval This article does not contain any studies with human participants performed by any of the authors.

\section{References}

He PR, Zhang WJ, Li Q (2001) A quadratic form-based approach to identification of kinematic chains in structural analysis and synthesis of mechanisms. In: ASME 2001 international design engineering technical conferences and computers and information in engineering conference, Pennsylvania, USA, Sep 2001, DETC2001/DAC-21067. 
He PR, Zhang WJ, Li Q (2003) A new method for detection of graph isomorphism based on the quadratic form. J Mech Design 125(3): 640-642.

He PR, Zhang WJ, Li Q (2005) Some further development on the eigensystem approach for graph isomorphism detection. J Frankl Inst-Eng Appl Math 342(6): 657-673.

Yang F, Deng ZQ, Tao JG, Li LF (2012) A new method for isomorphism identification in topological graphs using incident matrices. Mech Mach Theory 49: 298-307.

Madan SR (2014) Identification of isomorphism and detection of distinct mechanism of kinematic chains using invariant labeling of links. Int J Res Eng Tech 3(1): 17-23.

Pathapati VVNRPR, Rao AC (2002) A new technique based on loops to investigate displacement isomorphism in planetary gear trains. J Mech Des 124(4): 662-675.

Dharanipragada V, Chintada M (2016) Split Hamming string as an isomorphism test for one degree-offreedom planar simple-jointed kinematic chains containing sliders. J Mech Des 138(8): 1-8.

Rao AC, Pathapati VVNRPR (2000) Loop based detection of isomorphism among chains, inversions and type of freedom in multi degree of freedom chain. J Mech Des 122(1): 31-42.

Ding HF, Zhao J, Huang Z (2010) The establishment of edge-based loop algebra theory of kinematic chains and its applications. Eng Comput 26(2): 119-127.

Ding HF, Huang Z (2007) A new theory for the topological structure analysis of kinematic chains and its applications. Mech Mach Theory 42(10): 1264-1279.

Kong FG, Li Q, Zhang WJ (1999) An artificial neural network approach to mechanism kinematic chain isomorphism identification. Mech Mach Theory 34(2): 271-283.

Xiao RB, Tao ZW, Liu Y (2005) Isomorphism identification of kinematic chains using novel evolutionary approaches. J Comput Inf Sci Eng 5(1): 18-24.

Yang P, Pei ZH, Liao NB, Yang B (2007) Isomorphism identification for epicyclic gear mechanism based on mapping property and ant algorithm. Eng Comput 23(1): 49-54.

Yang P, Zeng KH (2009) A high-performance approach on mechanism isomorphism identification based on an adaptive hybrid genetic algorithm for digital intelligent manufacturing. Eng Comput 25(4): 397-403.

Zhang WJ, Li Q (1999) On a new approach to mechanism topology identification. J Mech Design 121(1): 57-64.

Arvind V, Kobler J, Kuhnert S, Rattan G, Vasudev Y (2015) On the isomorphism problem for decision trees and decision lists. Theor Comput Sci 590: 38-54.

Rai RK, Punjabi S (2018) Kinematic chains isomorphism identification using link connectivity number and entropy neglecting tolerance and clearance. Mech Mach Theory 123: 40-65.

Kamesh VV, Rao KM, Rao ABS (2017) An innovative approach to detect isomorphism in planar and geared kinematic chains using graph theory. J Mech Des 139(12): 1-11.

Dargar A, Hasan A, Khan RA (2011) A method for identification of isomorphism and structural properties of kinematic chains. Int J Mater Struct Integrity 5(4): 376-388.

Rai RK, Punjabi S (2018) A new algorithm of links labelling for the isomorphism detection of various kinematic chains using binary code. Mech Mach Theory 131: 1-32. 
Ding HF, Huang Z (2007) The establishment of the canonical perimeter topological graph of kinematic chains and isomorphism identification. J Mech Des 129(9): 915-923.

Gloria GM, Enrique MC, Domingo LR (2007) Improving neural networks for mechanism kinematic chain isomorphism identification. Neural Process Lett 26(2): 133-143.

Zou YH, He P (2016) An algorithm for identifying the isomorphism of planar multiple joint and gear train kinematic chains. Math Probl Eng 2016:1-15.

Yan HS, Hwang WM (1984) Linkage path code. Mech Mach Theory 19(4-5): 425-429.

Yadav JN, Pratap CR (1996) Mechanisms of a kinematic chain and the degree of structural similarity based on the concept of link-path code. Mech Mach Theory 31(7): 865-871.

Bedi GS, Sanyal S (2013) Loop based algorithm for automatic sketching of planar kinematic chains. In: Proceedings of the $1^{\text {st }}$ international and $16^{\text {th }}$ national conference on machines and mechanisms, $\mathrm{pp}$ : 452-456. 